\title{
DENSE SUBALGEBRAS OF PURELY INFINITE SIMPLE GROUPOID $C^{*}$-ALGEBRAS
}

\author{
JONATHAN H. BROWN, LISA ORLOFF CLARK, AND ASTRID AN HUEF
}

\begin{abstract}
A simple Steinberg algebra associated to an ample Hausdorff groupoid $G$ is algebraically purely infinite if and only if the characteristic functions of compact open subsets of the unit space are infinite idempotents. If a simple Steinberg algebra is algebraically purely infinite, then the reduced groupoid $C^{*}$-algebra $C_{r}^{*}(G)$ is simple and purely infinite. But the Steinberg algebra seems too small for the converse to hold. For this purpose we introduce an intermediate $*$-algebra $B(G)$ constructed using corners $1_{U} C_{r}^{*}(G) 1_{U}$ for all compact open subsets $U$ of the unit space of the groupoid. We then show that if $G$ is minimal and effective, then $B(G)$ is algebraically properly infinite if and only if $C_{r}^{*}(G)$ is purely infinite simple. We apply our results to the algebras of higher-rank graphs.
\end{abstract}

\section{INTRODUCTION}

Inspired by the success of classifying purely infinite simple $C^{*}$-algebras, Ara, Goodearl and Pardo extended the notion of purely infinite $C^{*}$-algebras to unital rings, and studied their basic properties [6]. Purely infinite rings were studied further in [5, 1, 19, 4]. In particular, [4 develops a general theory of purely infinite rings by presenting an algebraic parallel of the work in [21] on purely infinite $C^{*}$-algebras. Here we investigate a question asked in [4, Problem 8.4]:

Suppose that $A_{0}$ is a dense subalgebra of a $C^{*}$-algebra $A$. If $A_{0}$ is purely infinite in the algebraic sense, is $A$ purely infinite in the $C^{*}$-algebraic sense?

We focus on the situation where the $C^{*}$-algebra is a simple $C^{*}$-algebra associated to an ample Hausdorff groupoid, that is, an étale groupoid with a basis of compact open sets. Since every non-unital, purely infinite, simple, nuclear $C^{*}$-algebra in the UCT class is isomorphic to the reduced $C^{*}$-algebra $C_{r}^{*}(G)$ of some ample Hausdorff groupoid $G$ (see [35, p. 367]), this is a good setting to investigate this problem and its converse.

We investigate two different dense subalgebras of $C_{r}^{*}(G)$. The first is the complex Steinberg algebra $A(G)$ associated to an ample groupoid $G$. Simplicity of $A(G)$ and $C_{r}^{*}(G)$ are well understood (provided $G$ is amenable and second countable): $A(G)$ is algebraically simple if and only if $C_{r}^{*}(G)$ is $C^{*}$-algebraically simple, and this happens if

Date: February 2020.

2010 Mathematics Subject Classification. 46L05, 16S60.

Key words and phrases. Purely infinite ring, purely infinite $C^{*}$-algebra, ample groupoid, Steinberg algebra, Kumjian-Pask algebra, infinite projection, infinite idempotent.

Part of this research was done while the first named author was visiting the other two authors: he would like to thank them for their hospitality during this visit. This research was supported by the Marsden grant from the Royal Society of New Zealand and by the Seed grant IGRS03 from the University of Dayton Research Institute. We thank an anonymous referee for helpful suggestions, in particular for showing us Lemma 2.6 
and only if $G$ is minimal and effective [8]. For the remainder of the introduction, we assume that $G$ is a minimal and effective ample groupoid.

It was shown in [9, Theorem 4.1] that $C_{r}^{*}(G)$ is purely infinite if and only if, for every compact open set $U$ of the unit space, the characteristic function $1_{U}$ is an infinite projection. As a direct consequences, we obtain an affirmative answer to [4, Problem 8.4] when $A_{0}=A(G)$ (see Corollary 3.1). The converse remains an open question because, in a way, $A(G)$ is too small.

The second dense subalgebra $B(G)$ of $C_{r}^{*}(G)$ that we investigate contains the corners $1_{U} C_{r}^{*}(G) 1_{U}$ for all compact open subsets $U$ of the unit space of the groupoid, and so is much larger than $A(G)$. The reason $B(G)$ is useful is two-fold: First, unlike $C_{r}^{*}(G), B(G)$ is s-unital, and so we can apply the results of [4] directly to it. Second, unlike $A(G), B(G)$ has the property that $B(G) x B(G)$ is contained in $B(G)$ for every $x \in C_{r}^{*}(G)$. Using these two properties, we show in Theorem 4.7 that $B(G)$ is algebraically properly infinite if and only if $C^{*}(G)$ is purely infinite.

Our motivation for studying these dense subalgebras is to make progress on the longstanding open question of finding necessary and sufficient conditions on a groupoid $G$ that characterise when the $C_{r}^{*}(G)$ is purely infinite. Anantharaman-Delaroche introduced the notion of a locally contracting groupoid in [2, and then showed the reduced $C^{*}$-algebra of a locally contracting groupoid is purely infinite [2, Proposition 2.4]. It is not known if the converse holds, even for the $C^{*}$-algebras of higher-rank graphs which can be modelled using ample groupoids.

It follows from [2, Proposition 2.2] that in an ample, locally contracting groupoid, the $1_{U}$ are all infinite projections, but again it is not known if the converse holds. For the groupoids of higher-rank graphs, it would suffice to understand what graph properties ensure that all vertex projections are infinite projections in the graph $C^{*}$-algebra [9, Corollary 5.1].

Following [13, there has been substantial progress made in investigating a possible dichotomy for the $C^{*}$-algebras of ample groupoids [7, 29]: that $C^{*}$-algebras of ample groupoids are all either purely infinite or stably finite. At this stage, it has been established that the dichotomy holds when a certain type semigroup is almost unperforated. Also, Corollary 4.9 of [7] gives a sufficient condition for an inner exact groupoid to have a purely infinite $C^{*}$-algebra. Proposition 7.1 of [29] gives a necessary condition on $G$ for the $C^{*}$ algebra to be purely infinite. How either of these conditions relate to a locally contracting groupoid is not immediately clear.

The outline of this paper is as follows. In Section 2 we give some preliminaries, including algebraic and $C^{*}$-algebraic definitions of infinite idempotents/projections, and purely infinite rings and $C^{*}$-algebras. The main focus of Section 3 is to prove an algebraic version of [9, Theorem 4.1] for Steinberg algebras: $A(G)$ is algebraically purely infinite if and only if, for every compact open set $U$ of the unit space, $1_{U}$ is an infinite idempotent in $A(G)$. In Section 4, we introduce $B(G)$. Our main theorem, Theorem 4.7, says that $B(G)$ is algebraically properly infinite if and only if $C_{r}^{*}(G)$ is $C^{*}$-purely infinite. The forward implication is very general in that it does not require $G$ to be minimal or effective.

Finally, in Section 5, we show how our results apply to higher-rank graphs and their algebras. The Steinberg algebra of the graph groupoid is canonically isomorphic to the Kumjian-Pask algebra of the graph [3, 15], and the $C^{*}$-algebra of the graph groupoid is isomorphic to the $C^{*}$-algebra of the graph. We reconcile our results with recent results by Larki about purely infinite Kumjian-Pask algebras [23]. Finally, in Corollary 5.5, we 
consider the special case of an aperiodic and cofinal higher-rank graph which has a return path with an entrance, and show these graphs give rise to purely infinite algebras. Along the way, we prove Lemma 5.6 which allows us to construct return paths with entrances. This fills a gap in [16, Corollary 5.7] and [34, Proposition 8.8].

\section{PReliminaries}

We start by summarising the algebraic and $C^{*}$-algebraic definitions that we use from [4] and [21], respectively.

\section{Algebraic preliminaries.}

Definition 2.1. Suppose that $p$ and $q$ are idempotents in a ring $R, a \in R, x \in M_{k}(R)$ and $y \in M_{n}(R)$. Set $R a R:=\operatorname{span}\{c a d: c, d \in R\}$.

(1) $R$ is s-unital, if for each $r \in R$, there exist $u, v \in R$ such that $u r=r v=r$;

(2) $p \leq_{\text {ring }} q$, if $p q=q p=p$;

(3) $p<_{\text {ring }} q$ if $p \leq_{\text {ring }} q$ and $p \neq q$;

(4) $p \sim_{\text {ring }} q$ if there exist $s, t \in R$ such that $s t=p$ and $t s=q$;

(5) $x \precsim$ ring $y$ if there exists $\alpha \in M_{k n}(R)$ and $\beta \in M_{n k}(R)$ such that $x=\alpha y \beta$;

(6) $p$ is an infinite idempotent if there exists an idempotent $q$ such that $p \sim_{\text {ring }} q<_{\text {ring }} p$;

(7) $p$ is a algebraically properly infinite idempotent if there exists an idempotent $q$ such that $p \oplus p \sim_{\text {ring }} q \leq_{\text {ring }} p$;

(8) $a$ is algebraically infinite if there exists $t \in R \backslash\{0\}$ such that $a \oplus t \precsim$ ring $a$;

(9) $a$ is algebraically properly infinite if $a \neq 0$ and $a \oplus a \precsim_{\text {ring }} a$;

(10) $R$ is algebraically purely infinite simple if $R$ is algebraically simple ( $R$ has no nontrivial two-sided ideals) and every nonzero right ideal contains an infinite idempotent:

(11) $R$ is algebraically properly infinite (also called properly purely infinite) if every nonzero element of $R$ is algebraically properly infinite;

(12) $R$ is algebraically purely infinite if

(a) no quotient of $R$ is a division ring; and

(b) whenever $a \in R$ and $b \in R a R$, we have $b \precsim$ ring $a$.

Remarks 2.2. Here we clarify some of the relationships between the definitions given above.

(1) If $p$ and $q$ are idempotents with $p \leq_{\text {ring }} q$, then $p \precsim_{\text {ring }} q$.

(2) By [4, Remarks 2.7] an idempotent is an infinite idempotent if and only if it is algebraically infinite and it is an algebraically properly infinite idempotent if and only if it is algebraically properly infinite.

(3) Let $R$ be a ring with local units which means that for every finite set $F$ there exists an idempotent $e$ such that $F \subset e R e$. (The existence of local units implies that $R$ is s-unital.) Then $R$ is algebraically purely infinite simple if and only if (i) $R$ is not a division ring and (ii) for all $a, b \in R$ with $a \neq 0$ we have $b \precsim_{\text {ring }} a$ [1, Proposition 10]; in particular, $R$ is simple and algebraically purely infinite if and only if $R$ is algebraically purely infinite simple.

The next lemma is an algebraic version, for s-unital rings, of [21, Lemma 3.17].

Lemma 2.3. Suppose that $R$ is an s-unital ring. Let $p, q, a \in R$, and suppose that $q$ is an idempotent, and that $p$ is an infinite idempotent. 
(1) If $p \precsim_{\text {ring }}$ a, then a is algebraically infinite.

(2) If $p \leq_{\text {ring }} q$, then $q$ is an infinite idempotent.

(3) If $p \sim_{\text {ring }} q$, then $q$ is an infinite idempotent.

Proof. (11) is precisely [4, Lemma 3.9(iii)]. For (2), suppose that $p \leq_{\text {ring }} q$. By Remark 2.2(1), $p \precsim$ ring $q$. Since $p$ is algebraically infinite, (1) gives that $q$ is also algebraically infinite.

For (3), suppose $p \sim_{\text {ring }} q$. Then there exist $r, s \in R$ such that $r s=p$ and $s r=q$. Let $\alpha=r$ and $\beta=s$. Then

$$
\alpha q \beta=r q s=r(s r) s=(r s)^{2}=p^{2}=p .
$$

Now $p \leq_{\text {ring }} q$ and $p$ is algebraically infinite, and so $q$ is algebraically infinite by (1).

$C^{*}$-algebraic preliminaries. Let $A$ be a $C^{*}$-algebra. We write $A^{+}$for the positive elements of $A$, and then $A^{+}=\left\{a^{*} a: a \in A\right\}$. We write $C_{0}(X)$ for the $C^{*}$-algebra of continuous functions from $X$ to $\mathbb{C}$ that vanish at infinity. The following definitions come from [21].

Definition 2.4. Let $A$ be a $C^{*}$-algebra, let $p$ and $q$ be projections in $A$, and let $a, b \in A^{+}$, $x \in M_{k}(A)$ and $y \in M_{n}(A)$.

(1) $a \leq_{C^{*}} b$ if $b-a \in A^{+}$;

(2) $a<_{C^{*}} b$ if $a \leq_{C^{*}} b$ and $a \neq b$;

(3) $p \sim_{C^{*}} q$ if there exists $t \in A$ such that $t t^{*}=p$ and $t^{*} t=q$;

(4) $x \precsim C^{*} y$ if there exists a sequence $\left\{\alpha_{i}\right\} \subseteq M_{k, n}(A)$ such that $\alpha_{i} y \alpha_{i}^{*} \rightarrow x$ in norm;

(5) $p$ is an infinite projection if there exists a projection $q$ such that $p \sim_{C^{*}} q<_{C^{*}} p$;

(6) $p$ is a $C^{*}$-properly infinite projection if there exist mutually orthogonal projections $q_{1}$ and $q_{2}$ such that $q_{1} \leq p, q_{2} \leq p$ and $q_{1} \sim_{C^{*}} p \sim_{C^{*}} q_{2}$

(7) $a$ is $C^{*}$-infinite if there exists a nonzero $t \in A^{+}$such that $a \oplus t \precsim C^{*} a$;

(8) $a$ is $C^{*}$-properly infinite if $a \neq 0$ and $a \oplus a \precsim C^{*} a$;

(9) A $C^{*}$-subalgebra $B \subseteq A$ is hereditary if $a \leq_{C^{*}} b$ and $b \in B$ implies $a \in B$.

(10) $A$ is $C^{*}$-purely infinite simple if $A$ is simple ( $A$ has no nontrivial closed ideals) and every hereditary subalgebra contains an infinite projection;

(11) $A$ is $C^{*}$-purely infinite if

(a) there are no nonzero $*$-homomorphisms from $A$ to $\mathbb{C}$; and

(b) for every $a, b \in A^{+}$we have $b{ }_{C^{*}} a$ if and only if $b \in \overline{A a A}$.

Remarks 2.5. As in the algebraic situation there are relationships between these notions.

(1) By [21, Lemma 3.1], a projection $p$ is a $C^{*}$-infinite projection if and only if it is $C^{*}$-infinite. Similarly $p$ is a $C^{*}$-properly infinite projection if and only if $p$ is $C^{*}$-properly infinite.

(2) By Theorem 4.16 of 21, a $C^{*}$-algebra $A$ is $C^{*}$-purely infinite if and only if every nonzero positive element of $A$ is $C^{*}$-properly infinite. There is no algebraic analogue of [21, Theorem 4.16]; see [4, Examples 3.5] for a counter example.

(3) By [21, Proposition 5.4] a $C^{*}$-algebra $A$ that is simple and $C^{*}$-purely infinite is $C^{*}$-purely infinite simple. By [21, Proposition 4.7] a $C^{*}$-algebra $A$ is $C^{*}$-purely infinite simple implies that $A$ is simple and $A$ is $C^{*}$-purely infinite. Thus the two notions of $C^{*}$-purely infinite are compatible for simple $C^{*}$-algebras.

Lemma 2.6. Let $A$ be a $C^{*}$-algebra and $A_{0}$ a dense subalgebra. Let $p$ be a projection in $A_{0}$. If $p$ is an infinite idempotent in $A_{0}$, then $p$ is an infinite projection in $A$. 
Proof. Since $p$ is an infinite idempotent in $A_{0}$, there exists an idempotent $e \in A_{0}$ such that $p \sim_{\text {ring }} e<_{\text {ring }} p$. Thus $e A \subsetneq p A$. By [32, Exercise 3.11(1)] there exists a projection $q \in A$ such that $e \sim_{\text {ring }} q$ in $A$ and $e A=q A$. Since $\sim_{\text {ring }}$ is transitive, we have $p \sim_{\text {ring }} q$ in $A$. Thus we have $p \sim_{C^{*}} q$ by, for example, [32, Exercise 3.11(2)]. Also, since $p A \neq e A=q A$, we have $q \neq p$. To see that $q \leq_{C^{*}} p$, we show that $p q=q$, which suffices by [24, Theorem 2.3.2]. Since $q A \subsetneq p A$, there exists $a \in A$ such that $q^{2}=p a$, that is $q=p a$. So $p q=p^{2} a=p a=q$. Thus $p$ is an infinite projection.

Groupoids and groupoid algebras. Let $G$ be a topological groupoid. We write $G^{(0)}$ for the unit space of $G$, and $r, s: G \rightarrow G^{(0)}$ for the range and source maps. An open subset $B$ of $G$ is an open bisection if the source and range maps restrict to homeomorphisms on $B$, and $r(B)$ and $s(B)$ are open subsets of $G^{(0)}$. We say that $G$ is ample if $G$ has a basis of compact open bisections. Equivalently, $G$ is ample if $G$ is étale and its unit space $G^{(0)}$ is totally disconnected (see [17, Proposition 4.1]). In this paper, we consider only ample Hausdorff groupoids.

Let $G$ be an ample Hausdorff groupoid and let $K$ be a field. The Steinberg algebra $A_{K}(G)$ is the $K$-algebra of functions from $G$ to $K$ that are both locally constant and compactly supported. Addition is defined pointwise. The multiplication is convolution: for $f, g \in A_{K}(G)$ we have

$$
(f * g)(\gamma)=\sum_{\alpha \beta=\gamma} f(\alpha) g(\beta) .
$$

When $K$ has an involution $k \mapsto \bar{k}$, we define an involution $f \mapsto f^{*}$ on $A_{K}(G)$ by

$$
\left(f^{*}\right)(\gamma)=\overline{f\left(\gamma^{-1}\right)}
$$

We write $A(G)$ for $A_{\mathbb{C}}(G)$.

Let $B$ be a compact open bisection, and write $1_{B}$ for the characteristic function from $B$ to $K$. We will often use that every $f$ in $A_{K}(G)$ can be expressed as a sum

$$
f=\sum_{B \in F} c_{B} 1_{B}
$$

where $F$ is a finite set of disjoint compact open bisections and $0 \neq c_{B} \in K$ for each $B \in F$. For compact open bisections $B$ and $C$ we have

$$
1_{B} * 1_{C}=1_{B C} \quad \text { and } \quad 1_{B}^{*}=1_{B^{-1}} .
$$

Indeed, the compact open bisections in an ample groupoid form an inverse semigroup under the operations

$$
B C=\{\gamma \eta: r(\eta)=s(\gamma), \gamma \in B, \eta \in C\} \quad \text { and } \quad B^{-1}=\left\{\gamma^{-1}: \gamma \in B\right\}
$$

and the multiplication in $A_{K}(G)$ agrees with these operations. Every Steinberg algebra is s-unital by [10, Lemma 2.6]. See [11] and [36] for more information about Steinberg algebras.

The reduced groupoid $C^{*}$-algebra $C_{r}^{*}(G)$ was introduced in 31, and for ample groupoids the definition reduces to the following. With convolution and involution as above, the set $C_{c}(G)$ of continuous compactly supported function from $G$ to $\mathbb{C}$ is a *-algebra. Let $u \in G^{(0)}$. Set $G_{u}=\{\gamma \in G: s(\gamma)=u\}$. Write $\delta_{\gamma}$ for the basis of point masses in $\ell^{2}\left(G_{u}\right)$. 
There is a *-representation $R_{u}: C_{c}(G) \rightarrow \mathcal{B}\left(\ell^{2}\left(G_{u}\right)\right)$ such that, for $f \in C_{c}(G)$ and $\gamma \in G_{u}$,

$$
R_{u}(f) \delta_{\gamma}=\sum_{\{\alpha \in G: s(\alpha)=r(\gamma)\}} f(\alpha) \delta_{\alpha \gamma} .
$$

The reduced $C^{*}$-algebra $C_{r}^{*}(G)$ is the completion of the image of $C_{c}(G)$ under the direct sum $\bigoplus_{u \in G^{(0)}} R_{u}$. By construction, the complex Steinberg algebra $A(G)$ is a dense *subalgebra of $C_{r}^{*}(G)$.

We say that $G$ is effective if for every compact open bisection $B \subseteq G \backslash G^{(0)}$, there exists $\gamma \in B$ such that $s(\gamma) \neq r(\gamma)$. (See [8, Lemma 3.1] and [30, Corollary 3.3] for some equivalent characterisations of effective groupoids.) We say that $G$ is minimal if $G^{(0)}$ has no nontrivial open invariant subsets. By [14, Theorem 4.1], $A_{K}(G)$ is algebraically simple if and only if $G$ is minimal and effective. If $G$ is second-countable, minimal and effective, then $C_{r}^{*}(G)$ is $C^{*}$-simple, and the converse holds if $G$ is amenable (see, for example, [8, Theorem 5.1]).

Since $G$ is ample, the unit space $G^{(0)}$ is open in $G$, and we may view $C_{0}\left(G^{(0)}\right)$ as a subalgebra of $C_{r}^{*}(G)$. For $f, g \in C_{0}\left(G^{(0)}\right)$ we have

$$
f \leq_{C^{*}} g \text { if and only if } f(x) \leq g(x) \text { for all } x \in G^{(0)} .
$$

\section{Algebraically purely infinite simple Steinberg algebras}

By combining [9, Theorem 4.1] and Lemma 2.6, we get the following interesting result.

Corollary 3.1. Let $G$ be a second-countable ample Hausdorff groupoid. Suppose that $G$ is minimal and effective. If $A(G)$ is algebraically purely infinite, then $C_{r}^{*}(G)$ is $C^{*}$-purely infinite.

Proof. Since $G$ is minimal and effective, by [9, Theorem 4.1] it suffices to show that $1_{U}$ is an infinite projection for every compact open $U \subseteq G^{(0)}$. Since $A(G)$ is algebraically purely infinite simple, every idempotent is infinite [6, Proposition 1.5]. Thus every $1_{U}$ with $U \subseteq$ $G^{(0)}$ is an infinite idempotent in $A(G)$ and hence the result follows from Lemma 2.6.

We now prove the algebraic analogue of Theorem 4.1 in [9].

Theorem 3.2. Let $K$ be a field and let $G$ be an ample Hausdorff groupoid. Suppose that $G$ is minimal and effective. Then $A_{K}(G)$ is algebraically purely infinite simple if and only if $1_{U}$ is an infinite idempotent for every compact open $U \subseteq G^{(0)}$.

Proof. By [14, Theorem 4.1], $A_{K}(G)$ is algebraically simple if and only if $G$ is minimal and effective.

First suppose that $A_{K}(G)$ is algebraically purely infinite simple. Then all nonzero idempotents are infinite by [6, Proposition 1.5]. In particular, $1_{U}$ is an infinite idempotent for every compact open $U \subseteq G^{(0)}$.

Conversely, assume $1_{U}$ is an infinite idempotent for every compact open $U \subseteq G^{(0)}$. Fix a nonzero right ideal $I \subseteq A_{K}(G)$. We need to show that $I$ contains an infinite idempotent. Let $0 \neq b \in I$ and write

$$
b=\sum_{B \in F} c_{B} 1_{B}
$$


where $F$ is a finite set of disjoint compact open bisections and $0 \neq c_{B} \in K$ for each $B \in F$. Fix $B_{0} \in F$. Then

$$
c_{B_{0}}^{-1} b 1_{B_{0}^{-1}}=1_{r\left(B_{0}\right)}+\sum_{B \in F, B \neq B_{0}} c_{B_{0}}^{-1} c_{B} 1_{B B_{0}^{-1}} .
$$

Since $F$ is a disjoint collection, for each $B \neq B_{0}$ we have $B B_{0}^{-1} \subseteq G \backslash G^{(0)}$. So

$$
L:=\bigcup_{B \in F, B \neq B_{0}} B B_{0}^{-1}
$$

is a compact open subset of $G \backslash G^{(0)}$. Let $U:=r\left(B_{0}\right)$. Then $U$ is a compact open subset of $G^{(0)}$. Since $G$ is effective, we apply [8, Lemma 3.1(4)] to get a nonempty open set $V \subseteq U$ such that $V L V=\emptyset$. Now

$$
\begin{aligned}
1_{V}\left(c_{B_{0}}^{-1} b 1_{B_{0}^{-1}}\right) 1_{V} & =1_{V}\left(1_{r\left(B_{0}\right)}+\sum_{B \in F, B \neq B_{0}} c_{B_{0}}^{-1} c_{B} 1_{B B_{0}^{-1}}\right) 1_{V} \\
& =1_{V}+\sum_{B \in F, B \neq B_{0}} c_{B_{0}}^{-1} c_{B} 1_{V B B_{0}^{-1} V} \\
& =1_{V} .
\end{aligned}
$$

Set $p:=\left(c_{B_{0}}^{-1} b 1_{B_{0}^{-1}}\right) 1_{V}$. Then $p \in I$ and

$$
p^{2}=\left(c_{B_{0}}^{-1} b 1_{B_{0}^{-1}}\right)\left(1_{V}\left(c_{B_{0}}^{-1} b 1_{B_{0}^{-1}}\right) 1_{V}\right)=\left(c_{B_{0}}^{-1} b 1_{B_{0}^{-1}}\right) 1_{V}=p .
$$

Thus $p$ is a nonzero idempotent in $I$. Further, $1_{V} p=1_{V}$ and $p 1_{V}=p$, and hence $p \sim_{\text {ring }} 1_{V}$.

Corollary 3.3. Let $K$ be a field, let $G$ be an ample Hausdorff groupoid and let $\mathcal{B}$ be a basis for $G^{(0)}$ consisting of compact open subsets. Suppose that $G$ is minimal and effective. Then $A_{K}(G)$ is algebraically purely infinite simple if and only if $1_{U}$ is an algebraically infinite idempotent for every compact open $U \in \mathcal{B}$.

Proof. The forward implication is immediate from Theorem 3.2. For the reverse implication, suppose that $1_{U}$ is an algebraically infinite idempotent for every compact open $U \in \mathcal{B}$. Fix a compact open subset $V \subseteq G^{(0)}$. By Theorem 3.2 , it suffices to show $1_{V}$ is an infinite idempotent. Since $\mathcal{B}$ is a basis, there exists $W \in \mathcal{B}$ such that $W \subseteq V$. Then

$$
1_{W} 1_{V}=1_{W}=1_{V} 1_{W}
$$

and so $1_{W} \leq_{\text {ring }} 1_{V}$. By assumption, $1_{W}$ is an algebraically infinite idempotent. Since $A_{K}(G)$ is s-unital, Lemma 2.3(2) applies, and $1_{V}$ is an algebraically infinite idempotent as well.

A Hausdorff ample groupoid $G$ is locally contracting [2, Definition 2.1] if for every compact open $U \subseteq G^{(0)}$, there exists a nonempty compact open bisection $B \subseteq G$ such that

$$
s(B) \subsetneq r(B) \subseteq U .
$$

The following corollary to Theorem 3.2 is the algebraic analogue of [2, Proposition 2.4] which gives a useful sufficient condition for a $C^{*}$-algebra to be $C^{*}$-purely infinite simple.

Corollary 3.4. Let $K$ be a field and let $G$ be an ample Hausdorff groupoid. Suppose that $G$ is minimal, effective and locally contracting. Then $A_{K}(G)$ is algebraically purely infinite simple. 
Proof. Fix $U \subseteq G^{(0)}$. By Theorem 3.2, it suffices to show that $1_{U}$ is an infinite idempotent. Since $G$ is locally contracting, there exists a compact open bisection $B \subseteq G$ such that $s(B) \subsetneq r(B) \subseteq U$. Then

$$
1_{s(B)} 1_{r(B)}=1_{s(B)}=1_{r(B)} 1_{s(B)}
$$

gives $1_{s(B)} \leq_{\text {ring }} 1_{r(B)}$, and $s(B) \subsetneq r(B)$ gives $1_{s(B)}<_{\text {ring }} 1_{r(B)}$. Further,

$$
1_{B^{-1}} 1_{B}=1_{B^{-1} B}=1_{s(B)} \quad \text { and } \quad 1_{B} 1_{B^{-1}}=1_{B B^{-1}}=1_{r(B)}
$$

and so $1_{r(B)} \sim_{\text {ring }} 1_{s(B)}$. Thus

$$
1_{r(B)} \sim_{\text {ring }} 1_{s(B)}<_{\text {ring }} 1_{r(B)} .
$$

Therefore $1_{r(B)}$ is an infinite idempotent. Since $1_{r(B)} \leq_{\text {ring }} 1_{U}, 1_{U}$ is algebraically infinite by Lemma 2.3(2). By [4, Remarks 2.7], $1_{U}$ is an algebraically infinite idempotent as required.

\section{A LARGER DENSE SUBALGEBRA}

We want to connect the notion of $C^{*}$-purely infinite simple in the $C^{*}$-algebra with some kind of algebraic pure infiniteness. Corollary 3.1 is a start but we seek an if and only if characterisation and $A(G)$ seems too small. Even with the assumption that $G$ is minimal and effective, if $C_{r}^{*}(G)$ does not have an identity, then it is not s-unital and it is not algebraically simple: for example, the Pedersen ideal (see [27, Theorem 5.6.1]) is always a nontrivial dense nonclosed ideal.

We now introduce a large s-unital subalgebra of $C_{r}^{*}(G)$. Define

$$
B(G):=\left\{1_{U} x 1_{U}: x \in C_{r}^{*}(G) \text { and } U \subseteq G^{(0)} \text { is a compact open set }\right\} .
$$

It is straightforward to check that $A(G)$ is a subset of $B(G)$. Since the Pedersen ideal contains all the projections of $C_{r}^{*}(G)$ (see [27, 5.6.3]), it contains all elements of the form $1_{U}$ for compact open $U \subseteq G^{(0)}$. Thus $B(G)$ is contained in the Pedersen ideal.

We apply techniques from [4, Theorem 3.17] to see that when $G$ is minimal and effective, $B(G)$ is algebraically properly infinite if and only if $C_{r}^{*}(G)$ is $C^{*}$-purely infinite.

Lemma 4.1. Let $G$ be an ample Hausdorff groupoid. Then $B(G)$ is a dense s-unital subalgebra of $C_{r}^{*}(G)$.

Proof. That $B(G)$ is dense in $C_{r}^{*}(G)$ follows because $A(G) \subseteq B(G)$ and $A(G)$ is dense in $C_{r}^{*}(G)$ by [11, Proposition 4.2]. For any $1_{U} x 1_{U} \in B(G)$ we have $1_{U}\left(1_{U} x 1_{U}\right) 1_{U}=1_{U} x 1_{U}$, and so $B(G)$ is s-unital. To see that $B(G)$ is a subalgebra, fix $b_{1}:=1_{U_{1}} x 1_{U_{1}}$ and $b_{2}:=$ $1_{U_{2}} x 1_{U_{2}}$ in $B(G)$ and and $r \in \mathbb{C}$. Let $U:=U_{1} \cup U_{2}$. Then $U$ is compact and open because the $U_{i}$ are. Then

Similarly,

$$
b_{1}-b_{2}=1_{U}\left(1_{U_{1}} x 1_{U_{1}}-1_{U_{2}} x 1_{U_{2}}\right) 1_{U} \in B(G) .
$$

$$
b_{1} b_{2}=1_{U}\left(1_{U_{1}} x 1_{U_{1}} 1_{U_{2}} x 1_{U_{2}}\right) 1_{U} \in B(G) \quad \text { and } \quad r b_{1}=r 1_{U_{1}} x 1_{U_{1}}=1_{U_{1}}(r x) 1_{U_{1}} \in B(G) .
$$

Remark 4.2. If $G^{(0)}$ is compact, then $C_{r}^{*}(G)$ is unital with unit $1_{G^{(0)}}$. Thus for any $x \in C_{r}^{*}(G), x=1_{G^{(0)}} x 1_{G^{(0)}}$ and hence $B(G)=C_{r}^{*}(G)$.

Proposition 4.3. Let $G$ be a second-countable ample Hausdorff groupoid. Suppose that $G$ is minimal and effective. If $B(G)$ is algebraically properly infinite, then $C_{r}^{*}(G)$ is $C^{*}$ purely infinite. 
Proof. We show that $C_{r}^{*}(G)$ is $C^{*}$-purely infinite by verifying the two conditions of Definition 2.4(11). First, we have to show that there are no nonzero $C^{*}$-homomorphisms from $C_{r}^{*}(G)$ to $\mathbb{C}$. Aiming for a contradiction, suppose that there is a nonzero $C^{*}$ homomorphism $\pi: C_{r}^{*}(G) \rightarrow \mathbb{C}$. Since $G$ is minimal and effective, $C_{r}^{*}(G)$ is simple [31, Proposition II.4.6], so $\pi$ is injective, and $C_{r}^{*}(G)$ is $C^{*}$-isomorphic to $\mathbb{C}$. In particular, $A(G)$ is isomorphic to $\mathbb{C}$. But $A(G)$ is algebraically purely infinite, and hence contains an infinite idempotent. But then so does $\mathbb{C}$, a contradiction. Hence there are no nonzero homomorphisms from $C_{r}^{*}(G)$ to $\mathbb{C}$.

Second, we have to show that for $x, y \in C_{r}^{*}(G)^{+}$, we have $y \precsim C^{*} x$ if and only if $y \in \overline{C_{r}^{*}(G) x C_{r}^{*}(G)}$. Since $C_{r}^{*}(G)$ is simple, $\overline{C_{r}^{*}(G) x C_{r}^{*}(G)}=C_{r}^{*}(G)$; so it suffices to show that $y \precsim_{C^{*}} x$ for all $x, y \in C_{r}^{*}(G)^{+}$. Fix $x, y \in C_{r}^{*}(G)^{+}$. Since $B(G)$ is dense in $C_{r}^{*}(G)$, we have

$$
\overline{B(G) x B(G)}=\overline{C_{r}^{*}(G) x C_{r}^{*}(G)}=C_{r}^{*}(G) .
$$

Fix $\epsilon>0$. Since $y$ is a positive element, so is $\sqrt{y}$. Since $\sqrt{y} \in \overline{B(G) x B(G)}$, there exists a sequence $b_{n} \in B(G) x B(G)$ such that $b_{n} \rightarrow \sqrt{y}$. Since $B(G) B(G) x B(G) \subseteq B(G)$ we have

$$
b_{n} b_{n}^{*} \in B(G) x B(G) B(G) x B(G) \subseteq B(G) x B(G)
$$

and $b_{n} b_{n}^{*} \rightarrow y$. Thus there exists a positive $b \in B(G) x B(G)$ such that $\|b-y\|<\epsilon$. Now [21, Lemma 2.5(ii)] gives $(y-\epsilon)_{+} \precsim_{C^{*}} b$. Since $\epsilon$ was fixed, [21, Proposition 2.6] gives $y \precsim_{C^{*}} b$.

We claim that $b \precsim_{C^{*}} x$. By the definition of $B(G) x B(G)$, there exist $c_{i}, d_{i} \in B(G)$ such that $b=\sum_{i=1}^{k} c_{i} x d_{i}$, and hence there exists a compact open subset $W$ of $G^{(0)}$ such that $b=\sum_{i=1}^{k} 1_{W} c_{i} 1_{W} x 1_{W} d_{i} 1_{W}$. Thus $b \in B(G) 1_{W} x 1_{W} B(G)$. Since $B(G)$ is s-unital (by Lemma 4.1) and is algebraically properly infinite (by Proposition 4.6), it follows from [4, Lemma 3.4(i)] that $B(G)$ is algebraically purely infinite. Thus by Definition 2.1 (12b), $b \in B(G) \cap B(G) 1_{W} x 1_{W} B(G)$ implies $b \precsim_{\text {ring }} 1_{W} x 1_{W}$ in $B(G)$, that is, there exist $c, d \in B(G)$ such that

$$
b=c\left(1_{W} x 1_{W}\right) d=\left(c 1_{W}\right) x\left(1_{W} d\right) .
$$

Now applying [33, Proposition 2.4 (iii) $\Rightarrow$ (ii)] to the positive elements $b, x$ of $C_{r}^{*}(G)$ and the constant sequences $c 1_{W}, 1_{W} d$, gives a sequence $\left\{r_{n}\right\} \subseteq C_{r}^{*}(G)$ such that $r_{n} x r_{n}^{*} \rightarrow b$. Thus $b \precsim_{C^{*}} x$. Since $\precsim_{C^{*}}$ is transitive, we get $y \precsim_{C^{*}} x$.

Lemma 4.4. If $1_{U}$ is a $C^{*}$-properly infinite projection in $C_{r}^{*}(G)$, then $1_{U}$ is an algebraically properly infinite idempotent in $B(G)$.

Proof. Let $p:=1_{U}$ be an algebraically properly infinite projection in $C_{r}^{*}(G)$. By the definition of algebraically properly infinite projection, there exist $q_{1}, q_{2}, x, y \in C_{r}^{*}(G)$ such that

$$
x^{*} x=p, \quad x x^{*}=q_{1} \leq_{C^{*}} p, \quad y^{*} y=p, \quad \text { and } \quad y y^{*}=q_{2} \leq_{C^{*}} p-q_{1} .
$$

Thus $x$ and $y$ are partial isometries in $C_{r}^{*}(G)$ with range projections $q_{1}$ and $q_{2}$, respectively, and source projection $p$. Thus $x p=x$ and $q_{1} x=x$, and similarly for $y$. Take $x^{\prime}:=p x p$ and $y^{\prime}:=$ pyp. Then $x^{\prime}, y^{\prime} \in B(G)$. Now let $z$ be the 1 by 2 matrix $\left[x^{\prime} y^{\prime}\right]$. It is straightforward to check that

$$
z^{*} z=p \oplus p \quad \text { and } \quad z z^{*}=q_{1}+q_{2} \leq_{C^{*}} p .
$$

Thus $p$ is an algebraically properly infinite idempotent in $B(G)$. 
Lemma 4.5. Let $G$ be a minimal and effective groupoid. Suppose that $U, V \subseteq G^{(0)}$ are nonempty compact open sets such that $1_{U}$ is an algebraically properly infinite idempotent in $B(G)$. Then $1_{V} \precsim_{\text {ring }} 1_{U}$ in $B(G)$.

Proof. Since $G$ is minimal and effective, $A(G)$ is simple. Thus the ideal generated by $1_{U}$ in $A(G)$ is all of $A(G)$. Therefore there exist $a_{i}, b_{i} \in A(G)$ such that

$$
\begin{aligned}
1_{V} & =\sum_{i=1}^{n} a_{i} 1_{U} b_{i} \\
& \left.\precsim_{\text {ring }} \bigoplus_{i=1}^{n} a_{i} 1_{U} b_{i} \text { by [4, Lemma } 2.2(\text { vi })\right] \text { because } A(G) \text { is s-unital } \\
& \left.\precsim_{\text {ring }} \bigoplus_{i=1}^{n} 1_{U} \text { by [4, Lemma } 2.2(\mathrm{ii})\right] \\
& \precsim_{\text {ring }} 1_{U} \text { in } B(G)
\end{aligned}
$$

because $1_{U}$ is algebraically properly infinite in $B(G)$. Finally, since $\precsim_{\text {ring }}$ is transitive by [4, Lemma 2.2(i)] we have $1_{V} \precsim$ ring $1_{U}$ in $B(G)$.

Proposition 4.6. Let $G$ be a second-countable ample Hausdorff groupoid. Suppose that $G$ is minimal and effective, and that $C_{r}^{*}(G)$ is $C^{*}$-purely infinite. Then the algebra

$$
B(G)=\left\{1_{U} x 1_{U}: x \in C_{r}^{*}(G) \text { and } U \subseteq G^{(0)} \text { is a compact open set }\right\}
$$

of Lemma 4.1 is algebraically properly infinite.

Proof. Since $G$ is minimal and effective, $C_{r}^{*}(G)$ is $C^{*}$-purely infinite simple. Then [21, Theorem 4.16] says that every positive element of $C_{r}^{*}(G)$ is $C^{*}$-properly infinite. In particular, for every compact open $U \subseteq G^{(0)}, 1_{U}$ is a $C^{*}$-properly infinite projection in $C_{r}^{*}(G)$. So Lemma 4.4 says every $1_{U}$ is an algebraically properly infinite idempotent in $B(G)$.

Fix a nonzero $a \in B(G)$. We need to show that $a$ is algebraically properly infinite, that is, that $a \oplus a \precsim_{\text {ring }} a$. Since $a \in B(G)$, there exist a compact open $V \subseteq G^{(0)}$ and $x \in C_{r}^{*}(G)$ such that $a=1_{V} x 1_{V}$.

We claim that there exists a compact open $U \subseteq G^{(0)}$ such that $1_{U} \precsim$ ring $a^{*} a$ in $B(G)$. Since $a^{*} a$ is positive, by the proof of [9, Lemma 3.2] there exists a nonzero $h \in C_{0}\left(G^{(0)}\right)^{+}$ such that $h \precsim_{\text {ring }} a^{*} a$ in $C_{r}^{*}(G)$. (The statement of [9, Lemma 3.2] gives $h$ such that $h \precsim_{C^{*}} a^{*} a$, but a look at the proof shows $h \precsim_{\text {ring }} a^{*} a$.) Since $h \in C_{0}\left(G^{(0)}\right)^{+} \backslash\{0\}$ there exists a compact open subset $U$ of $G^{(0)}$ such that $h(u)>0$ for all $u \in U$. Define $g$ by

$$
g(u)= \begin{cases}\frac{1}{\sqrt{h(u)}} & \text { if } u \in U \\ 0 & \text { otherwise. }\end{cases}
$$

Then $g \in C_{0}\left(G^{(0)}\right)$, and $g h g=1_{U}$. Thus $1_{U} \precsim$ ring $h$ in $C_{r}^{*}(G)$. Now the transitivity of $\precsim_{\text {ring gives }} 1_{U} \precsim_{\text {ring }} a^{*} a$ in $C_{r}^{*}(G)$. In particular, there exists $y, z \in C_{r}^{*}(G)$ such that $z a^{*} a y=1_{U}$.

Let $W:=U \cup V$. Then $1_{U}=1_{W} 1_{U} 1_{W}$ and $a^{*} a=1_{V} x^{*} 1_{V} 1_{V} x 1_{V}=1_{W} a^{*} a 1_{W}$, and

$$
1_{W} z 1_{W} a^{*} a 1_{W} y 1_{W}=1_{W} z a^{*} a y 1_{W}=1_{W} 1_{U} 1_{W}=1_{U} .
$$

Thus $1_{U} \precsim$ ring $a^{*} a$ in $B(G)$, as claimed. 
We have $a^{*} a=a^{*} a 1_{V}$ and $a^{*}=1_{V} x^{*} 1_{V} \in B(G)$, and hence

$$
a^{*} a=a^{*} a 1_{V}=\left(1_{V} x^{*} 1_{V}\right) a\left(1_{V}\right) .
$$

Thus $a^{*} a \precsim$ ring $a$ in $B(G)$. Now $1_{U} \precsim_{\text {ring }} a$ in $B(G)$ by transitivity of $\precsim_{\text {ring }}$.

Since $B(G)$ is s-unital and $1_{U}$ an algebraically properly infinite idempotent in $B(G)$, we apply [4, Lemma 3.9(ii)] to get that

$$
a \oplus 1_{U} \precsim \text { ring } a
$$

in $B(G)$. We have $a \precsim$ ring $1_{V}$ because $a=\left(1_{V} x 1_{V}\right) 1_{V}\left(1_{V}\right)$ and Lemma 4.5 implies $1_{V} \precsim_{\text {ring }} 1_{U}$ in $B(G)$. The transitivity of $\precsim_{\text {ring implies } a} \precsim_{\text {ring }} 1_{U}$ in $B(G)$. We also have $a \precsim_{\text {ring }} a$ because $B(G)$ is s-unital, and so

$$
a \oplus a \precsim \text { ring } a \oplus 1_{U}
$$

by [4, Lemma 2.2(ii)]. Finally, transitivity gives $a \oplus a \precsim$ ring $a$ in $B(G)$.

Combining Propositions 4.3 and 4.6 we get our main theorem.

Theorem 4.7. Let $G$ be a second-countable ample Hausdorff groupoid. Suppose that $G$ is minimal and effective. Then $B(G)$ is algebraically properly infinite if and only if $C_{r}^{*}(G)$ is $C^{*}$-purely infinite simple.

Theorem 4.7 together with Corollary 3.1 give the following corollary.

Corollary 4.8. Let $G$ be an ample Hausdorff groupoid. Suppose that $G$ is effective and minimal. If $A(G)$ is algebraically purely infinite, then $B(G)$ is algebraically properly infinite.

The following remain open questions:

Questions 4.9. (1) Is there a groupoid $G$ such that $A(G)$ is algebraically purely infinite simple and $C_{r}^{*}(G)$ is not algebraically purely infinite?

(2) Is there a groupoid $G$ such that $C_{r}^{*}(G)$ is $C^{*}$-purely infinite simple and $A(G)$ is not algebraically purely infinite simple? (In other words, does the converse of Corollary 3.1 fail?).

(3) Let $U$ be a compact open subset of $G^{(0)}$. If $1_{U}$ is an infinite projection in $C_{r}^{*}(G)$, is it an infinite idempotent in $A(G)$ ? This would be a kind of converse to Lemma 2.6.

\section{KumJian-PASK ALGEBRAS}

In this section we show how our results apply to the subclass of algebras associated to higher-rank graphs. $C^{*}$-purely infinite simple $C^{*}$-algebras of higher-rank graphs are considered in [25, 34, 16, 13, 9, 26], but, in general, a condition on the graph that characterises pure infiniteness has remained elusive.

Kumjian-Pask algebras were defined in [3] for higher-rank graphs without sources, and were then generalised to locally convex graphs in [12] and to finitely aligned graphs in [15]. The groupoids built from $k$-graphs are described, for example, in [22, 18, 38, 20]. To make our exposition as self contained as possible we will include some of this construction below.

We view $\mathbb{N}^{k}$ as a category with one object and composition given by addition. A higherrank graph of rank $k$ or $k$-graph is a countable category $\Lambda$ with a functor $d: \Lambda \rightarrow \mathbb{N}^{k}$, called the degree map, that satisfies a unique factorisation property: 
if $\lambda \in \Lambda$ with $d(\lambda)=m+n$, then there exist paths $\mu, \nu \in \Lambda$ such that $d(\mu)=$ $m, d(\nu)=n$ and $\lambda=\mu \nu$.

We call the domain and codomain of a morphism $\lambda$ the source and range of $\lambda$, respectively, and write $s(\lambda)$ and $r(\lambda)$ for them. We call the objects and morphisms in $\Lambda$ vertices and paths respectively; we denote the set of vertices by $\Lambda^{0}$ and the set of paths by $\Lambda$. Thus $s, r: \Lambda \rightarrow \Lambda^{0}$. For $v \in \Lambda^{0}$ and $n \in \mathbb{N}^{k}$, we set $v \Lambda:=r^{-1}(v), \Lambda^{n}:=d^{-1}(n)$ and $v \Lambda^{n}:=v \Lambda \cap \Lambda^{n}$.

For $m=\left(m_{1}, \ldots, m_{k}\right)$ and $n=\left(n_{1}, \ldots, n_{k}\right) \in \mathbb{N}^{k}$, we define

$$
\begin{aligned}
& m \wedge n:=\left(\min \left\{m_{1}, n_{1}\right\}, \ldots, \min \left\{m_{k}, n_{k}\right\}\right) \text { and } \\
& m \vee n:=\left(\max \left\{m_{1}, n_{1}\right\}, \ldots, \max \left\{m_{k}, n_{k}\right\}\right) .
\end{aligned}
$$

Let $\lambda, \mu \in \Lambda$. If $d(\lambda)=m+n$, we write $\lambda(0, m)$ and $\lambda(m, m+n)$ for the unique paths of degree $m$ and $n$ such that $\lambda=\lambda(0, m) \lambda(m, m+n)$. Then $\tau \in \Lambda$ is a minimal common extension of $\lambda$ and $\mu$ if

$$
d(\tau)=d(\lambda) \vee d(\mu), \tau(0, d(\lambda))=\lambda \text { and } \tau(0, d(\mu))=\mu .
$$

We define

$\Lambda^{\min }(\lambda, \mu):=\{(\rho, \tau) \in \Lambda \times \Lambda: \lambda \rho=\mu \tau$ is a minimal common extension of $\lambda, \mu\}$.

Then $\Lambda$ is finitely aligned if $\Lambda^{\min }(\lambda, \mu)$ is finite (possibly empty) for all $\lambda, \mu \in \Lambda$. This class of $k$-graphs was introduced in [28] and is the most general type of $k$-graph considered in the literature.

A set $E \subseteq v \Lambda$ is exhaustive if for every $\lambda \in v \Lambda$, there exists $\mu \in E$ such that $\Lambda^{\min }(\lambda, \mu) \neq \emptyset$. The set of finite exhaustive sets is then

$$
\operatorname{FE}(\Lambda)=\bigcup_{v \in \Lambda^{0}}\{E \subseteq v \Lambda \backslash\{v\}: E \text { is finite and exhaustive }\} .
$$

The unit space of the groupoid of a finitely aligned higher-rank graph consists of the set of boundary paths, defined as follows. First, for $k \in \mathbb{N}$ and $m \in(\mathbb{N} \cup\{\infty\})^{k}$, let $\Omega_{k, m}$ be the category with objects $\left\{p \in \mathbb{N}^{k}: p \leq m\right\}$, morphisms

$$
\left\{(p, q): p, q \in \mathbb{N}^{k}, p \leq q \leq m\right\},
$$

$r(p, q)=p$ and $s(p, q)=q$. With degree functor $d(p, q)=q-p, \Omega_{k, m}$ is a $k$-graph. A degree-preserving functor $x: \Omega_{k, m} \rightarrow \Lambda$ is a boundary path of $\Lambda$ if for all $n \in \mathbb{N}^{k}$ with $n \leq m$ and all $E \in x(n, n) \mathrm{FE}(\Lambda)$, there exists $\lambda \in E$ such that $x(n, n+d(\lambda))=\lambda$. We write $\partial \Lambda$ for the set of all boundary paths.

For $p \in \mathbb{N}^{k}$ and $\lambda \in \Lambda^{p}$ we define $\sigma^{p}(\lambda)=\lambda(p, d(\lambda))$. As a set, the groupoid of a finitely aligned higher-rank graph is

$$
\begin{aligned}
G_{\Lambda}:=\{ & (x, m, y) \in \partial \Lambda \times \mathbb{Z}^{k} \times \partial \Lambda: \text { there exists } p, q \in \mathbb{N}^{k} \text { such that } p \leq d(x), q \leq d(y), \\
& \left.p-q=m \text { and } \sigma^{p}(x)=\sigma^{q}(y)\right\} .
\end{aligned}
$$

The range of $(x, m, y) \in G_{\Lambda}$ is $x$ and its source is $y$. Composition is given by

$$
(x, m, y)(y, n, z)=(x, m+n, z),
$$

and a computation shows that this is well-defined. We identify $\partial \Lambda$ with $G_{\Lambda}^{(0)}$ by $x \mapsto$ $(x, 0, x)$. 
When endowed with the topology described in [38], $G_{\Lambda}$ is a second-countable, Hausdorff and ample groupoid. For $\lambda \in \Lambda$ and finite subsets $F$ of $s(\lambda) \Lambda$, define

$$
Z(\lambda):=\{x \in \partial \Lambda: x(0, d(\lambda))=\lambda\} \quad \text { and } \quad Z(\lambda \backslash F):=Z(\lambda) \backslash\left(\bigcup_{\nu \in F} Z(\lambda \nu)\right) .
$$

Then the unit space $G_{\Lambda}^{(0)}$ has a basis of compact open sets of the form $Z(\lambda \backslash F)$. For each $\lambda, \mu \in \Lambda$ with $s(\lambda)=s(\mu)$, define

$$
Z(\lambda, \mu):=\{(\lambda x, d(\lambda)-d(\mu), \mu x): x \in \partial \Lambda \text { and } s(\lambda)=s(\mu)=r(x)\} .
$$

Then the basis for the topology on $G_{\Lambda}$ consists of sets of the form

$$
Z(\lambda, \mu \backslash F):=Z(\lambda, \mu) \backslash\left(\bigcup_{\nu \in F} Z(\lambda \nu, \mu \nu)\right)
$$

where again $F$ is a finite subset of $s(\lambda) \Lambda$. The subspace topology of $G_{\Lambda}^{(0)}$ is the one described above.

Given a field $K$, we define the Kumjian-Pask algebra and the $C^{*}$-algebra of $\Lambda$ by

$$
\operatorname{KP}_{K}(\Lambda):=A_{K}\left(G_{\Lambda}\right) \text { and } C^{*}(\Lambda):=C_{r}^{*}\left(G_{\Lambda}\right)
$$

respectively. These definitions are consistent with previous definitions in the literature via the isomorphism characterised by $s_{\mu} \mapsto 1_{Z(\mu, s(\mu))}$ ([15, Proposition 5.4] for $\operatorname{KP}_{K}(\Lambda)$ and [18, Theorem 6.9] for $\left.C^{*}(\Lambda)\right)$. To be consistent with the literature we write $s_{v}:=1_{Z(v, v)}$ and $s_{\mu}:=1_{Z(\mu, s(\mu))}$. We write $\operatorname{KP}(\Lambda)$ for $\operatorname{KP}_{\mathbb{C}}(\Lambda)$.

A $k$-graph $\Lambda$ is cofinal if for all $v \in \Lambda^{0}$ and $x \in \partial \Lambda$, there exists $n \leq d(x)$ such that $v \Lambda x(n)$ is nonempty. A $k$-graph is aperiodic if for all $v \in \Lambda^{0}$ there exists $x \in v \partial \Lambda$ such that $\alpha x=\beta x$ implies $\alpha=\beta$. By [15, Proposition 7.1] $G_{\Lambda}$ is minimal if and only if $\Lambda$ is cofinal, and by [15, Proposition 6.3] $G_{\Lambda}$ is effective if and only if $\Lambda$ is aperiodic.

Corollary 5.1. Let $\Lambda$ be a finitely aligned higher-rank graph. Suppose that $\Lambda$ is cofinal and aperiodic. Then $\mathrm{KP}(\Lambda)$ is algebraically purely infinite simple if and only if $s_{v}$ is an infinite idempotent for all $v \in \Lambda^{0}$.

Proof. Suppose that $\operatorname{KP}(\Lambda)$ is algebraically purely infinite simple. Fix $v \in \Lambda^{0}$. Then $Z(v)$ is a compact open subset of $\partial \Lambda=G_{\Lambda}^{(0)}$. Thus $s_{v}=1_{Z(v)}$ is an infinite idempotent by Theorem 3.2 .

Conversely, suppose that $s_{v}$ is an infinite idempotent for all $v \in \Lambda^{0}$. We consider the basis $\mathcal{B}=\{Z(\lambda \backslash F): \lambda \in \Lambda$, finite $F \subseteq s(\lambda) \Lambda\}$ of compact open sets for $G_{\Lambda}^{(0)}$, and seek to apply Corollary 3.3. First, consider a non-empty $Z(\lambda)$. Then we have

$$
1_{Z(\lambda)}=s_{\lambda} s_{\lambda^{*}} \sim_{\text {ring }} s_{\lambda^{*}} s_{\lambda}=s_{s(\lambda)} .
$$

By assumption, $s_{s(\lambda)}$ is an infinite idempotent. Since $\operatorname{KP}(\Lambda)$ is s-unital, $s_{\lambda} s_{\lambda^{*}}$ is an infinite idempotent by Lemma 2.3(3). It follows that $1_{Z(\lambda)}$ is an infinite idempotent in $\operatorname{KP}(\Lambda)$.

Second, consider a non-empty $Z(\lambda \backslash F)$. Then there exists $\lambda^{\prime} \in s(\lambda) \Lambda$ such that $Z\left(\lambda \lambda^{\prime}\right) \subseteq Z(\lambda \backslash F)$. Then

$$
1_{Z\left(\lambda \lambda^{\prime}\right)} \leq_{\text {ring }} 1_{Z(\lambda \backslash F)}
$$

and $1_{Z\left(\lambda \lambda^{\prime}\right)}$ is infinite by Lemma 2.3(2). Thus $\operatorname{KP}(\Lambda)$ algebraically purely infinite simple by Corollary 3.3 .

Corollary 5.2. Let $\Lambda$ be a finitely aligned higher-rank graph. Suppose that $\Lambda$ is cofinal and aperiodic. 
(1) If $\operatorname{KP}(\Lambda)$ is algebraically purely infinite simple, then $C^{*}(\Lambda)$ is $C^{*}$-purely infinite simple.

(2) Suppose that $s_{v}$ is an infinite idempotent in $\operatorname{KP}(\Lambda)$ for all $v \in \Lambda^{0}$. Then each $s_{v}$ is an infinite projection in $C^{*}(\Lambda)$.

Proof. (1) is immediate from Theorem 4.7.

(2) Since $s_{v}$ is an infinite idempotent in $\operatorname{KP}(\Lambda)$ for all $v \in \Lambda^{0}, \operatorname{KP}(\Lambda)$ is algebraically purely infinite simple by Corollary 5.1. Since $\mathrm{KP}(\Lambda)$ and $A\left(G_{\Lambda}\right)$ are isomorphic, Theorem 4.7 implies that $C^{*}(\Lambda)$ is $C^{*}$-purely infinite simple. Then every projection in $C^{*}(\Lambda)$ is an infinite projection by [9, Corollary 5.1].

We now connect our work with that of Larki in [23]. Recall from [16, Lemma 3.2] that a pair $(\mu, \nu)$ is called a generalised cycle if $Z(\mu) \subseteq Z(\nu)$. This generalised cycle has an entrance if the containment is strict.

Let $\Lambda$ be a finitely aligned, aperiodic and cofinal $k$-graph such that every vertex of $\Lambda$ can be reached from a generalised cycle with an entrance. Then [23, Theorem 5.4] implies that $\operatorname{KP}(\Lambda)$ is algebraically purely infinite simple. The next lemma shows that this also follows from Corollary 3.4 .

Lemma 5.3. Let $\Lambda$ be a finitely aligned higher-rank graph. Suppose that every vertex of $\Lambda$ can be reached from a generalised cycle with an entrance. Then $G_{\Lambda}$ is locally contracting.

Proof. Fix a nonempty compact open $U \subseteq G^{(0)}$. Then there exists $\lambda \in \Lambda$ and a finite subset $F$ of $s(\lambda) \Lambda$ such that $\emptyset \neq Z(\lambda \backslash F) \subseteq U$. Further, since every vertex can be reached from a generalised cycle, $Z(\lambda \backslash F) \neq\{\lambda\}$. Thus there exists $\lambda^{\prime} \in s(\lambda) \Lambda$ such that $d\left(\lambda^{\prime}\right) \neq 0$ and $Z\left(\lambda \lambda^{\prime}\right) \subseteq Z(\lambda \backslash F)$. By assumption, $s\left(\lambda^{\prime}\right)$ can be reached from a generalised cycle $(\mu, \nu)$ with an entrance. Say $\lambda \lambda^{\prime} \beta \mu \in \Lambda$. Then $\left(\lambda \lambda^{\prime} \beta \mu, \lambda \lambda^{\prime} \beta \nu\right)$ is a generalised cycle with an entrance. Then

$$
B=Z\left(\lambda \lambda^{\prime} \beta \mu, \lambda \lambda^{\prime} \beta \nu\right)
$$

is a compact open bisection with

$$
s(B)=Z\left(\lambda \lambda^{\prime} \beta \nu\right) \subsetneq Z\left(\lambda \lambda^{\prime} \beta \mu\right)=r(B) \subseteq U .
$$

Thus $G_{\Lambda}$ is locally contracting.

We now restrict to row-finite locally convex $k$-graphs. Let $\Lambda$ be a $k$-graph. Then $\Lambda$ is row-finite if $v \Lambda^{n}$ is finite for all $v \in \Lambda^{0}$ and $n \in \mathbb{N}^{k} ; \Lambda$ is locally convex if for every distinct $i, j \in\{1, \ldots, k\}, v \in \Lambda^{0}$ and paths $\lambda \in v \Lambda^{e_{i}}, \mu \in v \Lambda^{e_{j}}$, the sets $s(\lambda) \Lambda^{e_{j}}$ and $s(\mu) \Lambda^{e_{i}}$ are nonempty. Since our graphs might have sources, for $n \in \mathbb{N}$ we define

$$
\Lambda^{\leq n}:=\left\{\lambda \in \Lambda: d(\lambda) \leq n \text { and } s(\lambda) \Lambda^{e_{i}}=\emptyset \text { whenever } d(\lambda)+e_{i} \leq n\right\} .
$$

When $\Lambda$ is locally convex, by [37, Proposition 2.12] the boundary path space $\partial \Lambda$ equals

$$
\begin{aligned}
\Lambda^{\leq \infty}:= & \left\{x: \Omega_{k, m} \rightarrow \Lambda: x \text { is a functor such that if } v \in \Omega_{k, m}^{0} \text { and } v \Omega_{k, m}^{\leq e_{i}}=\{v\},\right. \\
& \text { then } \left.x(v) \Lambda^{\leq e_{i}}=\{x(v)\}\right\} .
\end{aligned}
$$

A path $\alpha \in \Lambda \backslash \Lambda^{0}$ is a return path if $r(\alpha)=s(\alpha)$; a return path $\alpha$ has an entrance if there exists a $\delta \in s(\alpha) \Lambda$ with $d(\delta) \leq d(\alpha)$ and $\alpha(0, d(\delta)) \neq \delta$. Our aim is to prove the following: 
Proposition 5.4. Let $\Lambda$ be a row-finite locally convex higher-rank graph. Suppose that $\Lambda$ is cofinal and aperiodic, and that $\Lambda$ contains a return path with an entrance. Then each vertex in $\Lambda$ can be reached from a return path with an entrance.

We then get:

Corollary 5.5. Let $\Lambda$ be a row-finite locally convex higher-rank graph. Suppose that $\Lambda$ is cofinal and aperiodic, and that $\Lambda$ contains a return path with an entrance. Then $\operatorname{KP}(\Lambda)$ and $C^{*}(\Lambda)$ are algebraically and $C^{*}$-purely infinite simple, respectively.

Proof. Let $\mu$ be a return path with an entrance. Then $(\mu \mu, \mu)$ is a generalised cycle with an entrance. Then Proposition 5.4 implies that every vertex can be reached from a generalised cycle. By [23, Theorem 5.4], $\mathrm{KP}(\Lambda)$ is algebraically purely infinite simple. Then $C^{*}(\Lambda)$ is $C^{*}$-purely infinite simple by Theorem 4.7 .

For 1-graphs, Proposition 5.4 follows directly from cofinality: if $\alpha$ is a return path with an entrance, then $x=\alpha \alpha \cdots$ is a boundary path, and cofinality implies that for every $v \in \Lambda^{0}$ there exists $\mu_{v} \in v \Lambda$ such that $s\left(\mu_{v}\right)$ is a vertex on $x$ and hence on $\alpha$. When we try to apply this idea to a general $k$-graph it fails: $x=\alpha \alpha \cdots$ may not be a boundary path. In fact, $x$ is not a boundary path whenever $d(\alpha) \wedge(1,1, \ldots, 1) \neq(1,1, \ldots, 1)$. The keys to proving Proposition 5.4 are Lemma 5.6 and Corollary 5.7, where we construct a boundary path $x$ that has enough return paths with entrances.

Lemma 5.6. Let $\Lambda$ be a row-finite and locally convex higher-rank graph. Suppose that $\Lambda$ has a return path $\alpha$ with an entrance and that there exists $\mu \in s(\alpha) \Lambda \backslash\{s(\alpha)\}$ with $d(\mu) \wedge d(\alpha)=0$. Then there exist $P \in \mathbb{N}$, a return path $\beta$ with an entrance such that $d(\beta)=P d(\alpha)$, and a path from $s(\beta)$ to $s(\alpha)$ of degree at least $d(\mu)$.

Proof. Let $\alpha$ be a return path with an entrance $\delta$. We may extend $\delta$ until $\delta \in s(\alpha) \Lambda \leq d(\alpha)$. By assumption there exists $\mu \in s(\alpha) \Lambda \backslash\{s(\alpha)\}$ with $d(\mu) \wedge d(\alpha)=0$.

By local convexity applied to the pair $(\mu, \delta)$, there exists $\mu_{0} \in s(\delta) \Lambda^{d(\mu)}$. If $s(\delta)=r(\delta)$ we can take $\mu_{0}=\mu$. We will now use the unique factorisation property to construct the path $\beta$.

Consider $\delta \mu_{0}$. Applying the unique factorisation property to the path $\delta \mu_{0}$ gives $\mu_{1} \in$ $s(\alpha) \Lambda^{d(\mu)}$ and $\gamma_{1} \in \Lambda^{\leq d(\alpha)} s\left(\mu_{0}\right)$ such that

$$
\delta \mu_{0}=\mu_{1} \gamma_{1} .
$$

By the unique factorisation property and induction, for $i \geq 2$ there exist $\mu_{i} \in s(\alpha) \Lambda^{d(\mu)}$ and $\gamma_{i} \in \Lambda^{d(\alpha)} s\left(\mu_{i-1}\right)$ such that

$$
\alpha \mu_{i-1}=\mu_{i} \gamma_{i}
$$

Notice that $\left\{\mu_{i}: i \geq 0\right\} \subseteq s(\alpha) \Lambda^{d(\mu)}$ is finite because $\Lambda$ is row-finite. So there exists $i \neq j$ such that $\mu_{i}=\mu_{j}$, and in particular $s\left(\mu_{i}\right)=s\left(\mu_{j}\right)$. Let $m, n \in \mathbb{N}$ with $m<n$ be such that $(m, n)$ is smallest with respect to the dictionary order and such that $\mu_{m}=\mu_{n}$. Then

$$
\theta:=\gamma_{n} \gamma_{n-1} \cdots \gamma_{m+1}
$$

is a return path.

First, suppose that $m \neq 0$. By the minimality of $(m, n), \gamma_{m} \neq \gamma_{i}$ for $m+1 \leq i \leq n$, and hence $\gamma_{m}$ is an entrance to $\theta$. Notice that $\delta \mu_{0} \gamma_{1} \ldots \gamma_{m}$ is a path of degree more than $d(\mu)$ from $s(\theta)$ to $s(\alpha)$. Since $d\left(\gamma_{i}\right)=d(\alpha)$ for $i \geq 2$, we get that $\beta:=\theta$ with $P=m-n$ satisfies the lemma. 
Second, suppose that $m=0$. Then

$$
\theta=\gamma_{n} \cdots \gamma_{1}
$$

Here $\theta$ is a return path, but it may not have an entrance. We will construct another return path with an entrance as required. Since $\mu_{n}=\mu_{0}$, we have $s(\alpha)=r(\delta)=s(\delta)$. In particular, $d(\delta)=d(\alpha)$ (for otherwise we could extend $\delta \in \Lambda \leq d(\alpha)$ further using $\alpha$ ).

We claim that there exist $q \in \mathbb{N}$ and $1 \leq i \leq n$ such that $\gamma_{i+q n} \neq \gamma_{i}$. Aiming for a contradiction, we suppose that $\gamma_{i+n q}=\gamma_{i}$ for all $q \in \mathbb{N}$ and $1 \leq i \leq n$. We will show by induction on $q$ that

$$
\Gamma_{q}:=\left\{\mu_{1}, \mu_{1+n}, \ldots, \mu_{1+q n}\right\}
$$

has $q+1$ distinct elements. Then $\Gamma_{q} \subseteq s(\alpha) \Lambda^{d(\mu)}$ for every $q$, and this contradicts that $s(\alpha) \Lambda^{d(\mu)}$ is finite. When $q=0$ we have $\Gamma_{q}=\left\{\mu_{1}\right\}$ and there is nothing to prove. For the inductive step, we will use the following two observations.

(1) $\mu_{1} \neq \mu_{1+t n}$ for any $t \in \mathbb{N}$ (for otherwise (5.1) gives $\alpha \mu_{t n}=\mu_{1+t n} \gamma_{1+t n}=\mu_{1} \gamma_{1}=$ $\delta \mu_{0}$, contradicting that $\alpha \neq \delta$ ).

(2) Let $1 \leq i \leq n$. Then (5.1) gives

$$
\mu_{i+1+t n} \gamma_{i+1}=\mu_{i+1+t n} \gamma_{i+1+t n}=\alpha \mu_{i+t n} .
$$

So if $\left\{\mu_{i+t n}: 0 \leq t \leq q\right\}$ has $q+1$ elements, then $\left\{\mu_{i+1+t n}: 0 \leq t \leq q\right\}$ has $q+1$ elements.

Now suppose that $q \geq 0$ and that $\Gamma_{q}$ has $q+1$ elements. We apply the observation (2) once to get that

$$
\left\{\mu_{2}, \mu_{2+n}, \ldots, \mu_{2+q n}\right\}
$$

has $q+1$ elements. After $n-1$ further applications of observation (2) we get that

$$
\left\{\mu_{1+q}, \mu_{1+2 q}, \ldots, \mu_{1+(q+1) n}\right\}
$$

has $q+1$ elements. Now we use observation (1) to see that

$$
\left\{\mu_{1}, \mu_{1+q}, \mu_{1+2 q}, \ldots, \mu_{1+(q+1) n}\right\}
$$

has $q+2$ elements. This proves our claim.

Let $\left(q_{0}, i_{0}\right)$ be smallest in the dictionary order such that $\gamma_{i_{0}+q_{0} n} \neq \gamma_{i_{0}}$. Since $\left\{\mu_{i}: i \geq\right.$ $\left.i_{0}+q_{0} n\right\}$ is finite, there exist smallest $(i, j)$ such that $i_{0}+q_{0} n \leq i<j$ and $s\left(\mu_{i}\right)=s\left(\mu_{j}\right)$. Then

$$
\beta:=\gamma_{j} \ldots \gamma_{i+1}
$$

is a return path of degree $(j-1) d(\alpha)$. If $i>i_{0}+q_{0} n$, then $\gamma_{i-1}$ is an entrance to $\beta$. If $i=i_{0}+q_{0} n$, then $\gamma_{i_{0}-1}$ is an entrance. In either case there is a path from $s(\beta)$ to $s(\alpha)$ of degree at least $d(\mu)$.

Corollary 5.7. Let $\Lambda$ be a row-finite and locally convex $k$-graph. Suppose that $\Lambda$ contains a return path with an entrance. Then there exists $x \in \Lambda \leq \infty$ such that for all $m \leq d(x)$ there exists $n \geq m$ and a $p \in \mathbb{N}^{k}$ such that $x(n, n+p)$ is a return path with an entrance.

Proof. Let $\alpha$ be a return path with an entrance. We construct a boundary path by induction. If $\alpha \alpha \cdots \in \Lambda^{\leq \infty}$ we are done. So suppose that $\alpha \alpha \cdots \notin \Lambda \leq \infty$. Let

$$
t=(1, \ldots, 1)-(1, \ldots, 1) \wedge d(\alpha) .
$$

Since $\alpha \alpha \cdots \notin \Lambda \leq \infty$, there exists $\mu_{1} \in s(\alpha) \Lambda \leq t$. Using Lemma 5.6 we find $P_{1} \in \mathbb{N}$ and a return path $\beta_{1}$ with an entrance such that, without loss of generality, $\beta_{1} \in s\left(\mu_{1}\right) \Lambda^{P_{1} d(\alpha)}$. 
Let $i \geq 1$, and suppose that we have $\mu_{i} \in \Lambda^{\leq t}, P_{i}$ and $\beta_{i} \in s\left(\mu_{i}\right) \Lambda^{P_{i} d(\alpha)} s\left(\mu_{i}\right)$ such that $\beta_{i}$ has an entrance. If $\beta_{i} \beta_{i} \cdots \in \Lambda \leq \infty$, then take $x=\beta_{i} \beta_{i} \cdots$. Otherwise there exists $\mu_{i+1} \in s\left(\beta_{i}\right) \Lambda^{\leq t}$. Using Lemma 5.6 applied to $\beta_{i}$ there exists $P_{i+1}^{\prime} \in \mathbb{N}$ and a return path $\beta_{i+1} \in s\left(\mu_{i+1}\right) \Lambda^{P_{i+1}^{\prime} P_{i} d(\alpha)}$ with an entrance. Take $P_{i+1}=P_{i+1}^{\prime} P_{i}$.

If this process terminates at $\beta_{i_{0}}$ take $x=\beta_{i_{0}} \beta_{i_{0}} \cdots$. If this process never terminates take

$$
x=\alpha \mu_{1} \beta_{1} \mu_{2} \beta_{2} \cdots .
$$

In either case, $x \in \Lambda \leq \infty$ has the desired properties.

Remark 5.8. Corollary [5.7 fills a gap in the proof of [16, Corollary 5.7] and in the last statement of [34, Proposition 8.8]. The last statement of [34, Proposition 8.8] claims that if $\Lambda$ is cofinal and contains a return path with an entrance, then every $v \in \Lambda^{0}$ can be reached from a return path with an entrance. Our Corollary 5.7 and cofinality ensure this is indeed the case. For [16, Corollary 5.7], Evans and Sims construct a single return path with entrance and then apply [34, Proposition 8.8].

Now we are ready to prove Proposition 5.4

Proof of Proposition 5.4. Since $\Lambda$ contains a return path with an entrance, Corollary 5.7 gives $x \in \Lambda \leq \infty$ such that for all $m \leq d(x)$ there exists $n \geq m$ and $p$ such that $x(n, n+p)$ is a return path with an entrance. Let $v \in \Lambda^{0}$. Since $\Lambda$ is cofinal there exists $m \leq d(x)$ such that $v \Lambda x(m)$ is nonempty. Pick $\omega_{v} \in v \Lambda x(m)$. Now pick $n \geq m$ and $p$ such that $x(n, n+p)$ is a return path with an entrance. Then $\mu_{v}=\omega_{v} x(m, n)$ connects $v$ to a return path with an entrance as desired.

\section{REFERENCES}

[1] G. Abrams and G. Aranda Pino, Purely infinite simple Leavitt path algebras, J. Pure Appl. Algebra 207 (2006), 553-63.

[2] C. Anantharaman-Delaroche, Purely infinite $C^{*}$-algebras arising from dynamical systems, Bull. Soc. Math. France 125 (1997), 199-225.

[3] G. Aranda Pino, J. Clark, A. an Huef and I. Raeburn, Kumjian-Pask algebras of higher-rank graphs, Trans. Amer. Math. Soc. 365 (2013), 3613-3641.

[4] G. Aranda Pino, K.R. Goodearl, F. Perera and M. Siles Molina, Non-simple purely infinite rings, Amer. J. Math. 132 (2010), 563-610.

[5] P. Ara, The exchange property for purely infinite simple rings, Proc. Amer. Math. Soc. 132 (2004), $2543-2547$.

[6] P. Ara, K.R. Goodearl and E. Pardo, $K_{0}$ of purely infinite simple rings, K-Theory 26 (2002), 69-100.

[7] C. Bönicke and K. Li, Ideal structure and pure infiniteness of ample groupoid $C^{*}$-algebras, Ergodic Theory Dynam. Systems 40 (2020), 3-63.

[8] J.H. Brown, L.O. Clark, C. Farthing and A. Sims, Simplicity of algebras associated to étale groupoids, Semigroup Forum 88 (2014), 433-452.

[9] J.H. Brown, L.O. Clark and A. Sierakowski, Purely infinite $C^{*}$-algebras associated to étale groupoids, Ergodic Theory Dynam. Systems 35 (2015), 2397-2411.

[10] L.O. Clark, R. Exel and E. Pardo, A generalised uniqueness theorem and the graded ideal structure of Steinberg algebras, Forum. Math. 30 (2018), 533-552.

[11] L.O. Clark, C. Farthing, A. Sims and M. Tomforde, A groupoid generalisation of Leavitt path algebras, Semigroup Forum 89 (2014), 501-517.

[12] L.O. Clark, C. Flynn and A. an Huef, Kumjian-Pask algebras of locally convex higher-rank graphs, J. Algebra 399 (2014), 445-474.

[13] L.O. Clark, A. an Huef and A. Sims, AF-embeddability of 2-graph algebras and stable finiteness of k-graph algebras, J. Funct. Anal. 271 (2016), 958-991. 
[14] L.O. Clark and C. Edie-Michell, Uniqueness theorems for Steinberg algebras, Algebr. Represent. Theor. 18 (2015), 907-916.

[15] L.O. Clark and Y.E.P. Pangalela, Kumjian-Pask algebras of finitely-aligned higher-rank graphs, J. Algebra 482 (2017), 364-397.

[16] D.G. Evans and A. Sims, When is the Cuntz-Krieger algebra of a higher-rank graph approximately finite-dimensional?, J. Funct. Anal. 263 (2012), 183-215.

[17] R. Exel, Reconstructing a totally disconnected groupoid from its ample semigroup, Proc. Amer. Math. Soc. 138 (2008), 2991-3001.

[18] C. Farthing, P. Muhly and T. Yeend, Higher-rank graph $C^{*}$-algebras: an inverse semigroup and groupoid approach, Semigroup Forum 71 (2005), 159-187.

[19] M.A. González-Barroso and E. Pardo, Structure of nonunital purely infinite simple rings, Comm. Algebra 34 (2006), 617-624.

[20] A. an Huef, S. Kang and I. Raeburn, Spatial realisations of KMS states on the $C^{*}$-algebras of higherrank graphs, J. Math. Anal. Appl. 427 (2015), 977-1003.

[21] E. Kirchberg and M. Rørdam, Non-simple purely infinite $C^{*}$-algebras, Amer. J. Math. 122 (2000), $637-666$.

[22] A. Kumjian and D. Pask, Higher rank graph $C^{*}$-algebras, New York J. Math. 6 (2000), 1-20.

[23] H. Larki, Purely infinite simple Kumjian-Pask algebras, Forum Math. 30 (2018), 253-268.

[24] G.J. Murphy, $C^{*}$-Algebras and Operator Theory, Academic Press, Inc., Boston, 1990.

[25] D. Pask, I. Raeburn, M. Rørdam and A. Sims, Rank-two graphs whose $C^{*}$-algebras are direct limits of circle algebras, J. Funct. Anal. 239 (2006), 137-178.

[26] D. Pask, A. Sierakowski and A. Sims, Unbounded quasitraces, stable finiteness and pure infiniteness, Houston J. Math. 45 (2019), 763-814.

[27] G.K. Pedersen, $C^{*}$-algebras and their automorphism groups, Academic Press, London, 1979.

[28] I. Raeburn, A. Sims and T. Yeend, The $C^{*}$-algebras of finitely aligned higher-rank graphs, J. Funct. Anal. 213 (2004), 206-240.

[29] T. Rainone and A. Sims, A dichotomy for groupoid $C^{*}$-algebras, Ergodic Theory Dynam. Systems 40 (2020), 521-563.

[30] J. Renault, Cartan subalgebras in $C^{*}$-algebras, Irish Math. Soc. Bulletin 61 (2008), 29-63.

[31] J. Renault, A groupoid approach to $C^{*}$-algebras, Lecture Notes in Mathematics, vol. 793, Springer, Berlin, 1980.

[32] M. Rørdam, F. Larsen and N.J. Lausten, An Introduction to K-theory for $C^{*}$-Algebras, London Mathematics Society Student Texts, Cambridge University Press, London, 2000.

[33] M. Rørdam, On the structure of simple $C^{*}$-algebras tensored with a UHF-algebra. II, J. Funct. Anal. 107 (1992), 255-269.

[34] A. Sims, Gauge-invariant ideals in the $C^{*}$-algebras of finitely aligned higher-rank graphs, Canad. J. Math. 58 (2006), 1268-1290.

[35] J. Spielberg, Graph-based models for Kirchberg algebras J. Operator Theory 57 (2007), 347-374.

[36] B. Steinberg, A groupoid approach to inverse semigroup algebras, Adv. Math. 223 (2010), 689-727.

[37] S.G. Webster, The path space of a higher-rank graph, Studia Math. 204 (2011), 155-185.

[38] T. Yeend, Groupoid models for the $C^{*}$-algebras of topological higher-rank graphs, J. Operator Theory 51 (2007), 95-120.

(J.H. Brown) Department of Mathematics, University of Dayton, 300 College Park DayTON, OH 45469-2316 USA

E-mail address: jonathan.henry.brown@gmail.com

(L.O. Clark and A. an Huef) School of Mathematics and Statistics, Victoria University of Wellington, P.O. Box 600, Wellington 6140, New Zealand

E-mail address: lisa.clark@vuw.ac.nz

E-mail address: astrid.anhuef@vuw.ac.nz 